\title{
Correction: Health and sustainability outcomes of vegetarian dietary patterns: a revisit of the EPIC-Oxford and the Adventist Health Study-2 cohorts
}

\author{
Gina Segovia-Siapco' ${ }^{1}$ Joan Sabaté ${ }^{1}$
}

Published online: 25 April 2019

(c) Springer Nature Limited 2019

\section{Correction to: European Journal of Clinical Nutrition https://doi.org/10.1038/s41430-018-0310-z published online 2 October 2018}

Since publication of the original version of this article, the authors noticed that some values of Table 4 were arranged under incorrect columns. These values have now been arranged correctly in both the HTML and PDF versions of this article.

Furthermore, a few textual changes were made in the Health outcomes of vegetarians section to align with the corrected values of Table 4 in both the HTML and PDF versions of this article.
Gina Segovia-Siapco

gsiapco@1lu.edu

1 Center for Nutrition, Healthy Lifestyle, and Disease Prevention, School of Public Health, Loma Linda University, Loma Linda, CA, USA 